\title{
Décadrages Décadrages
}

cinéma, à travers champs Cinéma, à travers champs

6 | 2005

Train et cinéma

\section{Du Rail à La Roue}

\section{François Albera}

\section{OpenEdition}

Journals

Édition électronique

URL : https://journals.openedition.org/decadrages/479

DOI : $10.4000 /$ decadrages. 479

ISSN : 2297-5977

\section{Éditeur}

Association Décadrages

\section{Édition imprimée}

Date de publication : 10 octobre 2005

Pagination : 22-35

ISBN : 978-2-9700582-2-9

ISSN : 2235-7823

Référence électronique

François Albera, «Du Rail à La Roue », Décadrages [En ligne], 6 | 2005, mis en ligne le 20 janvier 2014, consulté le 27 mars 2022. URL : http://journals.openedition.org/decadrages/479 ; DOI : https:// doi.org/10.4000/decadrages. 479

(B) Décadrages 
1 Abel Gance, Prisme, Gallimard, Paris, 1930, p. 176. Avec la réserve que l'on doit garder à l'esprit que ce texte publié en 1930 a été écrit après le tournage du film et sa réception et non au moment même (quoique interpolé dans le livre entre des textes tirés des "carnets de 1918" [p. 158] et un autre daté mai 1916mai 1917 [p. 176]) -, quoi qu'il doive éventuellement auxdits "carnets" de l'époque de la conception et du tournage, il est fortement marqué par la rétrospection puisque la référence à la femme aimée qui meurt en 1921 le traverse.
La roue (France, 1922) d'Abel Gance est au centre d'un curieux chassécroisé qui fait à la fois sa renommée, l'importance que ce film exerça et aussi son rejet, la détestation que certains lui vouèrent. Son importance tient à la place qu'il accorde, semble-t-il pour la première fois dans l'histoire du cinéma de cette façon, non seulement à la mécanique, à la machine - en l'occurrence ferroviaire - dont on montre les pièces en gros-plans, que l'on détaille, mais aussi à la cadence, au rythme de la machine comme modèle de montage. Cet aspect du film pourtant - dont s'engoue, on le sait, Fernand Léger, lui-même hanté dans sa peinture par de telles préoccupations - paraît au spectateur contemporain dominé, sur la longue durée de ce film, par deux autres niveaux, l'un narratif et quelque peu feuilletonesque, l'autre symbolique voire allégorique. Les passages dévolus à la mécanique de la locomotive (bielles, pistons, tubulures, chaudière, roues, rails, tender, crochet, etc.) et au monde ferroviaire se révèlent finalement peu nombreux en regard de l'histoire contée, celle d'un double inceste contrarié, et celle d'une "tragédie humaine» que "coiffe» une référence continuelle au destin à travers la symbolique de la Roue.

Il n'y a pas chez Gance de contradiction entre ces niveaux, ni de concessions ou de sacrifices à des formes acceptées qui seraient le véhicule d'un propos plus moderniste, novateur: il déclare lui-même - certes plusieurs années après le film, en 1930:

"Je cherche quelque motif mélodramatique et en même temps sujet éternel qui puisse utiliser un monde fait pour le cinéma, le monde des locomotives, des rails, des disques, des fumées... et par contraste un monde de neige, de sommets, de solitude; une symphonie blanche succédant à une symphonie noire. Faire marcher les catastrophes des sentiments et celle des machines de pair, aussi grandes, aussi élevées comme signification les unes que les autres; montrer l'ubiquité de tout ce qui bat; d'un cœur et d'un tiroir de vapeur. Le drame étant créé par l'extérieur, par les ambiances qui vont dégager peu à peu leur héros, à l'inverse du drame théâtral...»1 
S'il y a "contrebande» dans ce film, à la faveur de ce «motif mélodramatique», c'est non pour faire valoir ce "monde fait pour le cinéma» que sont les locomotives, rails, disques et fumées, mais pour élargir le propos "sur le plan humain" et "rejoindre peut-être ainsi" "sans que personne ne s'en doute, [s] es travaux ésotériques» $\mathbf{2}$.

A écouter Gance, mais il est vrai plus tard, son dessein ultime a été cette symbolique de la roue du destin dont le motif iconographique apparaît dans les cartons du film et que les citations littéraires (Kipling, Poe, Shakespeare, Sophocle...) reprennent. Lesquelles revêtaient pour le cinéaste une fonction importante comme il l'explique à Jean Mitry en 1924, affirmant qu'"avant de réaliser une œuvre exclusivement visuelle», il s'est attaché "à démontrer le rapport <image-texte), c'est-àdire à prouver le rayonnement de l'image autour de ces citations [...]. Ces citations [...] irradiaient leur puissance par ces images qui rayonnaient autour d'elles» $\mathbf{3}$.

Si La roue a généré bien des expériences ultérieures radicales, à commencer par le Ballet mécanique (France, 1924) de Léger - lequel inspira ensuite les passages sur l'écrémeuse de La ligne générale (URSS, 1929) par exemple -, elle le doit aux conditions de réception des films muets de cette époque: conditions d'exploitation du film en fonction du public visé, variables des copies présentées. L'usage inauguré par le CASA de Canudo de présenter des extraits de films, des morceaux séparés, distingués pour leur valeur formelle, la dissociation de la fable et de ces moments plastiques, rythmiques («séparer le style de l'anecdote»), ces projections de cinéma "pur» qui ont pris une valeur démonstrative (souvent liés à des conférences, des expositions: Tedesco les appellera "sélections") est un des principaux vecteurs de ce phénomène.

Pour le dire en trois mots, le film de Gance pourrait être déplié en ses trois niveaux par la seule énumération de ces trois titres successifs : le Rail, la Rose du rail, la Roue.

\section{La Peine des hommes}

Le chassé-croisé évoqué tout à l'heure ne concerne cependant pas que Gance. A l'origine du film, en effet, il y a un roman du monde du travail, Le rail de Pierre Hamp (1912), exemple de littérature "mécanicienne». Dans Prisme, Gance l'évoque ainsi:

"J'ai lu le Rail de Hamp, il y a déjà quelque temps. Tout ce que je puis me remémorer c'est qu'il y a dedans une admirable catastrophe de chemin de fer, d'une réelle composition musicale.»

On reviendra sur la filiation du roman au film - généralement omise ou sous-estimée (ainsi Roger Icart ne l'évoque-t-il que brièvement), mais il
2 Ibid.

3 "Le présent et l'avenir - Rêve et réalités", interview d'Abel Gance par Jean Mitry, in Comœdia illustré, $1^{\text {er }}$ mai 1924 (cité par Roger Icart, "Etude sur une longue copie teintée...", in 1895, spécial "Gance", p. 285). 
4 Pierre Hamp (1876-1962), auteur prolifique d'une œuvre d'une trentaine de volumes sous le titre général La peine des hommes a disparu des librairies et des rééditions après des décennies de succès public, peut-être en raison de son approche particulière de la société qu'il décrit par les métiers, les industries, les commerces, sans doute en raison du procès qui lui fut intenté à la Libération (il était inspecteur du travail et participa au régime de Vichy) et qu'il évoque dans un volume qui clôt son cycle, L'Eternel (Self, Paris, 1948). Ce cycle vaudrait pourtant, autant et souvent mieux que Les hommes de bonne volonté de Jules Romains, Les Thibaut de Roger Martin Du Gard ou La chronique des Pasquier de Georges Duhamel, comme fresque sociale de la France de la première moitié du $X X^{e}$ siècle.

5 Sergueï Tretiakov, "La biographie de l'objet", dans La littérature factuelle, Moscou, 1929 (traduit dans Action Poétique, $\mathrm{n}^{\circ} 54$, et repris dans S. Tretiakov, Dans le front gauche de l'art, Maspéro, Paris, 1977, p. 101). vaut la peine de s'attarder un instant sur la personnalité de Pierre Hamp et sur son œuvre4. C'est un écrivain "moderne» d'un genre particulier, à la fois essayiste, romancier (occasionnellement dramaturge) et, pourrait-on dire, "enquêteur». Il consacre ses textes aux métiers, aux corporations, à la production industrielle ou artisanale en un hymne protéiforme au travail dont il salue l'assomption mécanicienne contemporaine. En 1920, son essai, La victoire mécanicienne voit dans le perfectionnement technique, dans les valeurs de rapidité d'exécution, de précision, etc. la plupart des solutions aux problèmes du monde quels qu'ils soient: guerres, illettrisme, santé, hygiène, confort... sans ignorer les aberrations ou les crimes du système industriel (le travail des enfants par exemple). Cette foi dans la machine et l'industrie - chez cet ancien anarchiste devenu socialiste de tendance saint-simonienne, proche d'Albert Thomas durant la guerre, qui rompra avec la SFIO en 1928 après Monsieur Curieux - l'amènera à discuter et disputer plus d'une fois le monde ouvrier et les syndicats en dépit de son exaltation des corporations, des groupes humains au travail (ce qu'il appelle «l'âme corporative» dans Gueules noires). Travailleur lui-même (il débute comme apprenti pâtissier en 1891, travaille dans l'hôtellerie puis entre aux chemins de fer avant de devenir inspecteur du travail), c'est un autodidacte que la fréquentation de l'Université populaire projette dans l'action sociale puis la littérature. Dans Les métiers blessés (1919), il reprend une série d'articles publiés dans l'Humanité entre 1916 et 1917 ainsi que ses controverses avec l'Union corporative des ouvriers mécaniciens à qui il reprochait leur ignorance, leur manque de perspective d'ensemble. Dans Moteurs (1942), il décrit une grève menée par des militants communistes dans une usine d'aviation pendant le Front populaire dont il dénonce les desseins (diminution du temps de travail, baisse des cadences, demande de nationalisation) face aux enjeux du moment (montée de l'hitlérisme en Allemagne, perfectionnements techniques aux Etats-Unis). Sa démarche d'écriture, appuyée sur ses convictions, promeut une sorte d'objectivisme, de littérature de "reportage» - il réalise d'ailleurs pour la grande presse à plusieurs reprises des "enquêtes" (notamment sur les chemins de fer, les mines, la pêche, les filatures, la Bourse). Il fut ainsi, dans les années 1920, apprécié en URSS, élogié par le «factualiste» du LEF Serguéi Tretiakov qui appela ses romans «biographie de l’objet» $\mathbf{5}$. Ses textes sont nourris de chiffres, de pourcentages: date, poids, coût, production, vitesse, salaire, numéro d'ordre, place... Le monde des filatures comme le ferroviaire sont à cet égard idéaux pour lui - dans l'un et l'autre d'ailleurs règnent les machines "Compound». L'ensemble de la communauté des cheminots (avec leurs catégories bien précises et les 
différences qu'ils font entre eux), chacun à sa place, participe d'un vaste dispositif mécanique qui exige exactitude, précision à la demi-seconde d'un geste, fonctionnement sans défaut de la garde-barrière (Madame Buisine dans Le travail invincible, 1918) au chauffeur, au garde freins, au coltineur, au manœuvrier, à l'aiguilleur. Une mauvaise appréciation de l'un d'eux et tout est compromis, de même que le machiniste Rogeau

"de la 600-chevaux Compound mise en service le 30 août à l'usine de constructions mécaniques Hénon \& Cie, ouvrant le lundi 11 septembre, à 5 h55, la vanne de vapeur pour parvenir au temps d'embrayage de tous les métiers : 6 heures, à la pleine vitesse de 90 tours, entendit un tapement de bielle sur la glissière » 6 ,

s'avisant de ce que l'examen de la pièce défectueuse mettrait 1200 ouvriers et ouvrières au chômage, "ôterait le travail de 2400 mains", corrige par l'émeri et l'huile et une surveillance de 55 heures d'affilée le défaut et permet à la machine de continuer et avec elle toute l'usine.

L'hyperconscience d'être à sa place dans un tout où l'on n'est pourtant qu'un rouage, l'intériorisation du rythme imposé par les bielles, l'unité des gestes qui se complètent («leurs gestes emboutis, l'un rythmant l'autre, s'ajoutent en un seul geste»7), telle est la qualité qu'exalte Hamp chez l'ouvrier, décriant par contre l'assurance abstraite ou lointaine des chefs, inspecteurs, délégués de la direction soucieux avant tout, en cas de problème, d'incriminer les ouvriers et disculper la compagnie ou l'usine.

L'évocation de ces grands ensembles industriels et mécaniques ordonnés, réglés est pour lui l'épopée des temps modernes. Transports, circulations, réseaux sont ses maîtres mots:

"un réseau de chemin de fer est, en psychologie et en travaux, une œuvre plus énorme que les Pyramides d'Egypte [...]. Le transport est le premier des arts [...]. Une pancarte de la gare Saint-Lazare indiquait:

¿Chartres, la plus belle cathédrale.

12 trains rapides par jour .

Ce sont deux monuments du travail humain dont l'un va vers l'autre : le réseau et la cathédrale. » $\mathbf{8}$

Hamp a exprimé plusieurs fois sa conception de l'art qu'il veut, en un temps où se lève "dans le socialisme la plus haute des mystiques: la mystique de l'idée de justice»(Les métiers blessés ${ }^{9}$ ), promis «lui aussi» à une révolution. Car «l'art contemporain» ne fait que

«recherche[r] sénilement les jeux pornographiques. Aimons la saine ruée qui en accomplira la dévastation. [...] Cessant de reproduire la gesticulation des oisifs et d'inventer la psychologie des rentières, l'art
6 Pierre Hamp, Le travail invincible, NRF, Paris, 1918, p. 198.

7 Pierre Hamp, Les métiers blessés, NRF, Paris, 1919 , p. 160.

8 Pierre Hamp, "Enquêtes", Gueules noires, Gallimard, Paris, 1938, pp. 220-221.

9 Les Métiers blessés, op. cit., p. 159. 
10 ld., p. 160. Voir également sa contribution à l'Encyclopédie française (dir. Lucien Febvre): "La littérature, image de la Société" (vol. XVII, 16.64-1/4, août 1935).

11 Pierre Hamp, Il faut que vous naissiez de nouveau, Gallimard, Paris, 1935.

12 Pierre Hamp, Mes métiers, NRF, Paris, 1929.

13 Pierre Hamp, Il faut que vous naissiez à nouveau, op. cit., pp. 286-287.

14 Voir Michel Collomb, La Littérature Art Déco. Sur le style d'époque, Klincksieck, Paris, 1987.

15 On rapporte dans Cinéa-Ciné pour tous, $\mathrm{n}^{\circ} 12 \mathrm{du} 1^{\text {er }}$ mai 1924 , la conférence d'Epstein au CASA, "L'Elément photogénique" du 11 avril 1924 au Théâtre Raymond Duncan. II y présente "ses Photogénies", c'est-à-dire un montage, destiné à cette seule circonstance dont "la première partie [...] reproduit simplement une machine à papier. Nous entendîmes pour la première fois applaudir des rouages. C'est qu'une profonde émotion esthétique se dégageait de cette vie intime de la machine mise à nu par la projection animée et remarquablement rendue, au point que les meules tournantes donnaient une impression subjective d'écrasement. [...] La seconde partie de la bande était empruntée à la fameuse foire de Cœur fidèle. L'orchestre, qui avait suivi avec une précision mathématique les rythmes de la machinerie, se déchaîna alors sur des airs de fête populaire, rajeunis par des accords de jazz. Ce fut un synchronisme des plus frappant que l'on ne confondit à aucun moment avec une inutile et puérile imitation de bruits..." va retrouver les temps où il était la sublimation du travail, du travail de la terre, du travail de la guerre. Le drame de l'usine est sur le même plan que L'Iliade. Les hommes qui aujourd'hui tiennent dans leur main la réalité, qui endurent le choc de la pierre qui tombe et de la machine qui éclate, sont des poètes à bouche close. Une harmonie tragique est dans leur souffrance inconnue. [...]

Dans la résistance au vertige, devant les sorcelleries du feu où suent les faces des durs peigneurs dont la flamme agile mord le corps perdu, tous ceux-là sont les hommes qui portent la peine du monde. Dans leur esprit vit la religion millénaire dont le Messie n'est pas encore venu : la Justice. Leur rêve est de l'espérer, leur passion est de l'établir. Ils sont capables pour elles d'aimer la mort. Quel esprit plus puissant peut donner à l'art son inspiration?»10

Dans Il faut que vous naissiez de nouveau $\mathbf{1 1}$, il raconte son itinéraire (déjà entamé dans Mes métiers, où il est pâtissier 12), sa formation (l'Université populaire), son travail aux chemins de fer et sa démission, devenu chef de gare, pour être écrivain mais fort de cette connaissance des métiers :

"quelle philosophie de bibliothèque valait cette expérience pour la formation des caractères? [...] L'homme nouveau que je cherchais en moi n'était pas tellement celui qui se libérerait du travail mais celui qui pourrait l'aimer. »13

On voit donc que cet écrivain attaché aux motifs et aux valeurs de la modernité les met en jeu tout autrement que les protagonistes du «style Arts Déco» dont Paul Morand est le parangon ${ }^{\mathbf{1 4}}$.

Quant au cinéma, Hamp s'exprime à son propos à plusieurs reprises et il est l'auteur d'un scénario.

En 1925, il participe aux conférences du Vieux Colombier de Tedesco par un exposé, "Photogénie du monde mécanique», qu'accompagne un montage de Grémillon, "Photogénie mécanique». On n'a pas conservé ce montage qui s'apparente manifestement à celui (perdu également) que réalise Epstein à la même époque: filmage de machines, de pièces en action, enchaînements des actions, sérialisation des mouvements, etc. Le film de Deslaw, La marche des machines (France, 1929), appartient sans doute à cette même série dont on a maint exemples partiels dans des films soviétiques (Eisenstein, Vertov) ou allemands (Ruttmann) $\mathbf{1 5}$.

Que dit Hamp dans cette conférence? Faute de disposer du texte même, on a deux indices de sa position sur le cinéma: un article de ParisJournal en août 1924 où il dénonce les feuilletons cinématographiques: "le cinéma oublie trop souvent sa véritable destination qui est la vie et la reproduction de la vie. Il n'est pas fait pour ressusciter de vieux 
feuilletons qui déshonorèrent jadis le rez-de-chaussée des journaux populaires et qui sont bien morts, définitivement oubliés.» et surtout son texte de La nouvelle fortune (1926) qui offre dix pages sur le cinéma sous le titre: "Le cinématographe contre la littérature».

S'il déplore là aussi que le cinématographe "parv[î]nt vite à l'affabulation", qu'il «adopt[â] t les habitudes de la grossièreté littéraire et se f[î]t remorquer par le mélodrame», voué aux «impostures» et à «l'aliénation mentale »16, il n’en dégage pas moins une positivité du cinéma. Comme le fera Edgar Morin dans Le cinéma ou l’homme imaginaire (1956), Hamp rattache le cinéma au rêve d'Icare: dans le délire d'irréalisme des diverses mythologies, «le seul essai mécanique est celui d'Icare. Il ne s'est pas contenté de rêver, il a construit. Par le mythe icarien, la poésie devient réalisatrice. Elle conçoit la machine.» Le génie du travail a maintenant dépassé la nature: «toutes les puissances de la création vont être humiliées par la mécanique», l'électricité, l'automobile, la télégraphie, etc.

«Mais le travail humain a construit ce que les poètes n'avaient pas osé concevoir: la mécanique même du rêve. Le cinématographe est une machine à songes. Il dépasse les mots, il va permettre de penser en images : l'enseignement comme l'amusement seront changés par le cinéma: ni l'école ni le théâtre ne peuvent repousser sa technique.» L'autre aspect de son propos est celui de l'art de masse (les foules l'adorent, «la masse du peuple prodigue à cet art de l'image mouvante un enthousiasme dont on ne trouve dans l'histoire du spectacle que deux exemples: les Mystères et Guignol»), l'art universel («langue internationale»), l'ubiquité («le monde entier devient présent à qui ne peut le parcourir»), le pouvoir médiatique («aux grandes cérémonies, il suffit de porter le trépied et le moulin pour que chacun fasse place. L'homme qui va écrire avec des images est plus puissant que la police.»).

Le film dont il écrit le scénario, réalisé par René Hervil en 1924, s'appelle Paris. La ville comme entité, organisme dont les habitants sont les éléments, ne peut qu'intéresser cet auteur. Il publiera d'ailleurs ultérieurement un album de photos de Kertesz sur Paris commentées par lui-même.

A l'annonce du scénario, la revue Cinéa-Cinépour tous se félicite que «l'un de nos jeunes écrivains les plus caractéristiques de la littérature moderne... l'auteur de La peine des hommes...» vienne de la sorte légitimer le cinéma; la revue insiste à quelques numéros de là sur le fait que René Hervil, metteur en scène de Paris s'attaquera aussitôt après à Knock de Jules Romains.

Or ces deux écrivains sont un peu plus que des littérateurs reconnus qui contribueraient à sortir le cinéma de sa marge culturelle. Jules
16 Pierre Hamp, La nouvelle fortune, NRF, Paris, 1926, pp. 246-247. 
17 Kino-fot, n 4, 1922.

18 Cinéa-Ciné pour tous, $n^{\circ} 18,1^{\text {er }}$ août 1924.
Romains avec son roman Donogoo Tonka (1920), avait l'ambition de fonder un genre nouveau, le récit cinématographique. Le texte donnera lieu à une adaptation théâtrale puis filmique mais d'après sa réécriture pour la scène (!). Traduit en russe en 1922, il aura une importance certaine dans les milieux d'avant-garde soviétiques: Ehrenbourg fondateur avec Lissitzky de l'Internationale constructiviste se réfère à lui, l'imite. La revue Kino-fot écrit à son sujet: "La littérature a enfin compris le nouveau rythme de la vie que seul le cinéma percevait jusqu'ici»17. L'année même où Hamp écrit Paris, Jacques Feyder tourne L'image également d'après Jules Romains tandis que sort sur les écrans L'autre Aile d'Andréani d'après le roman de Canudo.

Le film de Hamp-Hervil, «en face de la Butte où l'on s'amuse», veut, lui, montrer «la Butte où l'on peine, la montagne latine où s'abritent les labeurs scientifiques et les rêves des poètes, le Paris industriel, les usines, Javel, Billancourt... Il offrira de la ville un défilé de tableaux panoramiques et kaléidoscopiques. »18

Quel en est le scénario? Sur la Butte Montmartre, Jean Fleury, avant de se rendre à l'usine de 12000 ouvriers où il est mécanicien, étudie. Il espère en effet reprendre des études que la ruine de ses parents saignés par un requin de la haute finance, lui a fait interrompre. Sa fiancée, Aimée, sa voisine, est ouvrière dans un atelier de couture. François Roullet, un savant qui fut le professeur de Jean, vient, quant à lui, de faire une découverte sur la combustion de l'air qui va révolutionner la locomotion. C'est Maurice Revoil, le patron de Jean, sorti du peuple, qui est chargé de construire ce nouveau moteur. Jean y travaille chez lui pour ne pas ébruiter la découverte, en liaison TSF avec l'usine. Mais un banquier véreux, Alpéroff, vole des plans à Roullet et, pour les compléter, cherche à soudoyer Jean. Celui-ci résiste, une bagarre s'ensuit sur un échafaudage d'où Jean est précipité dans le vide. Alpéroff veut alors voler les plans de la machine mais celle-ci, laissée sans surveillance, s'emballe et explose, tuant le voleur... Jean s'est miraculeusement rattrapé dans sa chute et adoubé par le savant, aimé par sa fiancée, il a réussi son ascension sociale.

Tant que le film est en écriture - le scénario imaginé par Hamp est adapté par René Jeanne, qui en tirera un «roman dramatique» aux éditions Tallandier la même année - on en attend beaucoup en raison de la personnalité de l'écrivain.

Après sa sortie les commentaires sont divisés. Certains chroniqueurs se félicitent de la part documentaire du film laissant la vedette à Paris tandis que d'autres saluent le fait que le drame se dégage du document. Emile Vuillermoz salue «l'effort estimable de propagande et de 
pédagogie morale et sociale» mais déplore que Hamp «qui semblait devoir apporter dans nos studios l'œil neuf d'un artiste venu de l'extérieur... ait sacrifié aux pires traditions du cinéma»19.

Hamp s'est donc montré plus timide en cinéma qu'en littérature alors même qu'on créditait son style de proximité avec le film... Il n'est évidemment que scénariste et encore faudrait-il mesurer la part de René Jeanne dans cet affadissement du thème imaginé. Hervil qui n’a pas le talent de Gance a, de surcroît, filmé platement cette histoire laquelle, en dépit de ses rebondissements mélodramatiques (la fiancée est détournée du côté du luxe et de la haute couture puis revient vers Jean), eût pu donner lieu à des «morceaux de bravoure» autour de la vie de l'usine, des machines et de la présence de la ville comme entité collective. Tout au plus le film dispose-t-il à intervalles réguliers des parties documentaires sur la ville, les quartiers - filmés par André Sauvage.

\section{L'autre aile}

Il y a un troisième mouvement d'aller-retour autour de La roue qui concerne cette fois Ricciotto Canudo. En effet le critique et théoricien de l'esthétique du cinéma signe un roman "d'après La roue» chez Ferenczi en 1923. Nous n'allons pas développer ici l'analyse de ce texte paradoxal l'ayant fait ailleurs ${ }^{20}$, mais il est l'occasion de repérer une étonnante contradiction entre les déclarations d'intention du romancier, et du critique de La roue et le texte qu'il livre qui se révèle plus mélodramatique encore que le film... Canudo semble dans sa préface embrasser l'approche du Rail (mouvements collectifs, machine...) et il développe, en fait, la dimension Rose du rail du scénario.

Comme l'indique la liste des autres romans parus ou à paraitre de l'auteur, celui-ci paraît s'inscrire dans une perspective assez voisine de celle de Hamp. «Les Romans des foules nouvelles»: La ville sans chef, 1910-1920; Les libérés (Mémoires d'un aliéniste), 1911; Les transplantés (La ville Visage-du-Monde), 1913; L'autre aile (Roman de l'aviation), 1922. Selon Canudo, la "première vision du film» qu'eut Gance est celle d'

«une vie immense [qui grouille] autour des chemins de fer, et non seulement la vie des cheminots, mais celle des millions d'êtres que le train comme un geste implacable du destin des distances, remue, brise ou allonge à travers les espaces. Tout un monde de passion et d'action tourbillonne autour d'un simple train en marche, capable d'émouvoir le talent évocateur d'un Pierre Hamp. Ce monde a aussi ému Abel Gance.»21
19 Article collationné sans référence dans le fonds Rondel de la BNF.

20 Voir F. Albera, "La Roue roman de Ricciotto Canudo d'après la Roue d'Abel Gance", Colloque d'Udine, mars 2005 (à paraître).

21 La Roue. Roman d'après le Film d'Abel Gance par Canudo, Editions J. Ferenczi \& fils, Paris, 1923. 
22 Paris-Midi du 23 février 1923 (repris dans le Siècle du 24 février et in fine dans L'usine aux images, tant la première édition que la réédition due à Jean-Paul Morel).

23 Emile Vuillermoz, "Un film d’Abel Gance la Roue", Cinémagazine, no 8, 23 février 1923, pp. 329-331, et no 9, 2 mars 1923, pp. 363-366.
Cette vision, continue-t-il,

«répond à la nécessité esthétique la plus moderne qui pousse quelques artistes et quelques écrivains à représenter les remous de la psychologie des foules. Ils veulent remplacer la figuration artistique de la passion individuelle par l'évocation des collectivités...»

Cette définition correspond parfaitement à l'entreprise de Pierre Hamp ; elle n'est cependant adoptée ni dans le film ni dans le roman. Là où Hamp fait sourdre les destinées individuelles du collectif qui les détermine, Canudo donne la prééminence à la "psychologie des foules". Canudo a cité Hamp, il cite ensuite Paul Adam «[le] plus grand romancier des foules modernes" qui a préfacé ses Libérés -, et il oppose, dans sa préface, les «romanciers que satisfait l'analyse de l'individu en proie aux passions de l'amour» à d'autres écrivains qui "construisent aujourd'hui la synthèse de plusieurs ou de nombreuses âmes que transforme un courant de pensée». Comme Walt Whitman et Emile Verhaeren, on a chez Adam «la collectivité agissant comme un seul individu» et "la large poésie de la machine», une littérature peut-être "qui triomphera demain de toute la littérature d'alcôve ou de cabaret, dites pompeusement, l'une 〈psychologique〉 et l'autre 〈sociale〉».

Il cite enfin la Bête humaine de Zola. Le «mérite» de Gance, est-il dit, "est d'avoir impérieusement apporté à l'écran» le lyrisme de ces écrivains. Cependant ce "mérite», écrit Canudo, a une limite, celle... du cinéma. Dans sa critique de La roue, il écrit en effet du film:

"Une sorte d'exaltation folle chez tous les personnages, chacun isolé dans sa violence ou dans son rêve, semble leur venir par instants, de l'ambiance brutale de fer et de feu dont ils participent. Dans le roman écrit d'après le film, cette exaltation endémique explique la psychologie, les mouvements, les décisions des trois plans humains que l'écraniste a présentés avec la magie de la vision directe: la maison de Sisif, la vie des cheminots qui l'entourent, enfin, 〈l'âme de l'acier et du feu〉 qui règle tout le grouillement des énergies et des appétits. Dans le film la vision de l’Homme et celle de la Machine ne concordent pas toujours et la première apparaît souvent faible et incohérente au milieu de l'autre parfois sublime. »22

On retrouve là l'approche d'Emile Vuillermoz dans Cinémagazine: "La Roue est un film double: l'un sentimental, romanesque, feuilletonesque, puéril, l'autre est une «suite rythmique de l'acier» rayonnant une sensibilité qui centuple celle de l'homme et oriente le Cinéma vers des voies impérieusement nouvelles, étrangères à la littérature, à la peinture et même à la musique. »23 
Puis Canudo se réfère au montage de fragments de La roue montré au CASA et au Salon d'Automne qu'il baptisa "la Chanson du rail», "la Danse des roues", proposition approchant sans doute ce «Rythme pur» qu'il évoque en fin d'article quand il écrit, se référant à Moussinac (qui écrit sur La roue en ce sens dans Comoedia [19 janvier 1923] puis dans le Crapouillot [mars 1923]), que le film permet de tirer une «leçon»:

"Si l'on pouvait grouper tous les fragments épars dans La roue où les figures monstrueuses de l'acier, où les géants et les gnômes de la Mécanique entrent violemment dans le pauvre drame du cœur humain, en règlent la pulsation et en dominent toute la joie et la douleur, M. Abel Gance nous offrirait des pages singulièrement fortes sinon de Musique pure certes de Rythme pur. [...] Ce serait parfait.»

Mais ça ne l'est pas et, paradoxalement, la réécriture canudienne s'en éloigne encore.

\section{Le rail}

Revenons au livre Le rail dont Gance est parti pour entreprendre sa Roue car on mesurera de la sorte la différence que l'on peut faire entre le livre et le film sur la question de l'inscription du ferroviaire dans une fiction.

Hamp, on l'a dit, évoque avant tout une corporation, un milieu, un métier. Dans Le rail, il s'agit des chemins de fer (qu'il évoque également en tant que tels dans Gueules noires, dans Gens, dans Il faut que vous naissiez à nouveau) dont il a eu une connaissance directe (il est devenu chef de gare à Calais en 1906). Les personnages individués, nommés, brièvement caractérisés par leur allure physique, leur caractère, leur comportement, n'adviennent qu'en fonction des nécessités des opérations décrites. Ils ne se dégagent en rien, ni ne dominent une situation, ils sont à leur place, apparaissent et disparaissent. Dans Le rail la description du travail des cheminots connaît un événement, un déraillement. Puis un second, une grève. On voit donc qu'il ne s'agit que de mouvements ou de phénomènes collectifs. Toute évocation d'un personnage ou d'un groupe de travailleurs, est assortie d'indications sur leur salaire, leurs horaires, la nature calculable de leur travail. Ainsi:

«Cent cantonniers arrivaient en file hérissée de pinces, de pioches et de pelles. Lherminé, seize ans de service, dix-neuf cent francs, commandait la besogne des auxiliaires à 0,35 l'heure, encadrés par les commissionnés qui débutaient à 93 francs par mois et en touchaient 85, déduction faite du $5 \%$ pour leurs vieux jours ou ceux des autres. Les favorisés ajoutaient le profit d'un passage à niveau dont leur 
24 Pierre Hamp, Le rail, NRF, Paris, 1912 (éd. 1935, p. 18). femme poussait les barrières. Cela leur valait le logement et 30 francs par mois sur les grandes lignes, 15 sur les petites.» $\mathbf{2 4}$

Le film s'ouvre sur l'accident de chemin de fer qui avait tant impressionné Gance dans le livre.

Celui-ci se déroule pourtant très différemment chez Hamp que chez Gance : dans Le rail, cet accident intervient après 80 pages de description du fonctionnement de l'ensemble et le lecteur familiarisé avec les gestes des ouvriers ou les ordres des chefs, comprend comment un dysfonctionnement peut survenir. Chez Gance il introduit le film sans que l'on en sache les causes mais il est la matrice de la fiction: Sisif, le conducteur habitant près des voies, venu porter secours aux blessés, recueille la petite Norma, la «rose du rail». En effet sur ce fond de catastrophe, d'incendie, de wagons tordus, de voyageurs hébétés courant dans tous les sens, surgit la figure hiératique de Sisif cadré frontalement en plan rapproché et donnant des ordres. Voici l'enchaînement proposé par Hamp:

«L'aiguilleur Drahé, ouvrant le volet de sa cabine 1, cornait 5. Le train 129 s'annonçait. Fermant selon son habitude le signal 22, l'aiguilleur manœuvrait les leviers 17, 14, 11 et se tenait les deux mains sur le 21, prêt à le rabattre à l'évacuation des lames de l'aiguille (b) par le fourgon.

Il s'impatientait à l'obligation imminente d'écrire au rapport: ‘Arrêt du 129 au signal 22> et pensait :

¿Vingt sous de retenue de prime. Ce n'est pas moi qui écoperait de ça!)

On entendait grandir le sifflet du rapide qui engagea la courbe à pleine pression de sa Compound. Tous les manœuvriers, les deux bras levés, firent au train le signal d'arrêt. L'aiguille 21a, en pointe sur voie de service, retournée, la vitesse de cette masse aurait pu continuer sur l'espace libre. L'enclenchement l'empêchait. Etabli contre la faillibilité des hommes, il inutilisait leur initiative; le calcul, réalisé en fer, donnait la direction de la catastrophe. L'impuissance de rien changer dominait tout. Une tempête d'épouvante passait dans l'âme des manœuvriers. M. Legendre, magnifique en son agilité de professeur gymnaste, bondissait au-devant du rapide, les bras hauts, la bouche active à crier. Les wagons en obstacle sur 5 , enfin enlevés côté Nord, le 4922 avança lentement. L'espoir frais souffla. Le fourgon de queue dégageait la pointe 21b. Drahé, ses dix doigts pâlis, crispés au levier humide par sa sueur soudaine, le renversait à se casser les reins. Trop tard. L'aiguille manœuvrée sous la locomotive l'envoyait hors des rails. Le craquement du matériel désaligné, dansant sur le ballast, remplaça le glissement régulier des roues sur 
l'acier uni. Les cent vingt tonnes de fer de la Compound dont rien ne guidait plus la vitesse, choquaient à 80 kilomètres à l'heure, les cailloux lancés en mitraille sous les wagons de triage, puis la A.3609 se couchant sur les rails tordus offrit butoir au train qui s'écrasa sur elle en une forte détonation. Le brisement des bois et le ploiement des fers s'achevaient dans l'emmêlement des voitures, dont les débris cherchaient l'appui. Le dernier craquement monta mourir dans l'espace paisible où habitait le sourire du ciel. Le silence revint durer le temps qu'il faut aux hommes pour passer de leur habitude à l'épouvante illimitée. Puis les cris grandirent, rejoints par de nouveaux cris poussés à briser les gorges. Des voitures restées debout sur rails, toutes portières ouvertes, émettaient des fuyards qui se frappaient, tombaient dans la poussière créée par le choc. [...]

- Allo !... Le 129 déraillé en gare... Oui, le 129... Vous êtes sourd ?... Merde!

Les poches d'eau, vidées dans le foyer de la 3609, l'éteignaient... [...] - Le 129 dans le sable. On ramasse les morts!

Le mécanicien de la machine Sud répétait les trois coups du signal d'alarme; cette cadence de polka régularisait le pas gymnastique des employés de la P.V., des secrétaires du chef ballottant la civière d'où sortaient trois mois de poussière et des accrocheurs Nord chargés de la boîte de secours. [...]

- Allons! il faut en sortir!

Une aussi prompte et complète révolution des habitudes donnait aux choses du métier une défiguration redoutable. M. Qualin ne savait plus par quel bout les prendre. [...] Concentré sur l'idée de continuation du trafic, il étudiait le bouleversement des choses pour y maintenir le plus possible d'habitudes.» $\mathbf{2 5}$

La séquence la plus "mécanicienne" de La roue est sans doute celle dite de «la mort de Norma Compound» où Sisif, perdant la vue, emballe sa machine et veut la jeter contre un obstacle, plutôt que de la voir confiée à son ennemi Jacobin. Comme on peut le voir ci-dessous en lisant le découpage qu'en a établi Roger Icart d'après une copie d'époque ${ }^{\mathbf{2 6}}$, cette séquence multiplie - après montage car la confrontation avec le scénario montre qu'il en allait différemment - les inserts très brefs de «bielles, ballast» ou autres éléments mécaniques ou ferroviaires (rails, fumée, régulateur, cadrans) qui viennent articuler les dimensions d'accélération et d'inéluctabilité de la course engagée. L'alternance avec les gros-plans de Sisif, l'accent sur son regard en particulier, son sourire, etc. rattache cependant tout cet engrenage à la maîtrise «suicidaire» du conducteur : il n'est pas un élément du dispositif machinique, il n'est pas emporté
25 ld., pp. 92-100.

26 Roger Icart, Abel Gance ou le Prométhée foudroyé, L'Age d'Homme, Lausanne, 1983, pp. 151-156. Voir l'annexe. 
par la machine, il la conduit. Il n'est guère de ces plans de paysage qui défile, rails, bielles, etc. qui ne soient rapportés au regard de Sisif. C’est pourquoi la fin de l'épisode: sa déploration, le dialogue avec la machine éventrée et expirante redonne toute sa place à l'écriture métaphorique et allégorique du film. Comparons ce passage avec la partie finale du Cuirassé Potemkine, dite de «l'attente de l'escadre " où se succèdent manomètres, volants, etc. mais où les marins, çà et là individués, font partie d'un dispositif d'ensemble parfaitement réglé.

Cette ambiguïté gancienne par rapport à la mécanique se retrouve dans les choix qu'il fait de plans fixes, frontaux de Sisif (manifestement filmés à l'arrêt) montés parmi ces plans pris depuis une machine en marche et qui d'ailleurs se répètent plus qu'ils n'inscrivent un mouvement évolutif de la marche. C'est paradoxalement le seul montage - syntaxe qui répond à une certaine mécanicité et qui «accélère» ses composants (raccourcissement, répétition) envisagés presque abstraitement.

\section{Annexe}

sous-titre: "La Mort de , Norma Compound,."

2433 - Plan d'ensemble. La locomotive devant la prise d'eau, Sisif à la conduite; Mâchefer à terre s'installe sur le volant d'ouverture et tire un livre de sa poche-revolver.

2434 - Plan rapproché. Sisif à l'intérieur de la cabine de conduite. II regarde à l'extérieur puis, d'un geste vif, tire le régulateur.

2435 - Plan d'ensemble. La locomotive part. Le tuyau d'eau dégagé asperge Mâchefer qui saute à bas du volant, ahuri.

2436 - Plan rapproché. Sisif esquisse un sourire et tire à fond le régulateur.

2437 - Plan d'ensemble. Mâchefer court à la poursuite de la locomotive. Un cheminot vient fermer la vanne.

2438 - Plan rapproché. Sisif vu de $3 / 4$ dos.

2439 - Plongée à la verticale. Bielles en action, ballast qui défile.

2440 - Plan rapproché. Sisif vu de 3/4 dos.

2441 - Plan d'ensemble. La locomotive passe à vive allure dans un nuage de fumée. Mâchefer court derrière.

2442 - Plongée à la verticale. Bielles. Ballast.

2443 - Plan rapproché. Sisif se retourne. Regard intense.

2444 - Plongée à la verticale. Bielles. Ballast.

2446 [sic] - Plan rapproché. Sisif de profil. II regarde intensément.

2447 - Plan d'ensemble. La lomotive passe en deuxième voie. Mâchefer cesse de courir, puis revient en arrière.

2448 - Plan rapproché. Sisif regarde anxieusement vers l'arrière.

2449 - Plan rapproché. Une cabine d'aiguillage, vue de l'extérieur. Mâchefer arrive par la droite et s'adresse à l'aiguilleur, tout en regardant vers la direction prise par Sisif. L'aiguilleur apparaît à la fenêtre.

sous-titre: "Sisif vient de partir comme un fou sur la 7. Quelle aiguille a-t-il?"

2450 - Suite du même plan. L'aiguilleur regarde au loin.
2451 - Plan d'ensemble. La locomotive disparaît au loin dans un nuage de fumée.

2452 - Plan rapproché. Cabine d'aiguillage. L'aiguilleur regarde à l'intérieur, puis se retourne vers Mâchefer.

sous-titre (sur fond de rails qui défilent): "Y a qu'lui qui s'cassera la gueule, il est sur le garage à butoir 22."

2453 - Suite du même plan. Mâchefer, furieux et désorienté, s'en va.

2454 - Plan rapproché. Sisif.

2455 - Plongée. Bielles en action.

2456 - Plan rapproché. Mâchefer de face regarde intensément.

2457 - Plan rapproché. L'aiguilleur regarde.

2458 - Plan américain. Sisif dans la cabine (vu du tender).

2459 - Plan rapproché. L'aiguilleur regarde.

2460 - Plan américain. Sisif dans la cabine (vu du tender).

2461 - Plongée. Bielles en action.

2461 bis - Paysage qui défile (depuis la cabine).

2461 ter - Rails qui défilent.

2463 - Plongée. Bielles en action.

2464 - Gros plan Sisif vu de face. Visage tendu.

2464 bis - Rails qui défilent.

2464 ter - Plan moyen. Locomotive vue de face sortant d'un tunnel. Travelling arrière.

2465 - Gros plan Sisif vu de face.

2465 bis - Plan moyen locomotive vue de face. Travelling arrière.

2465 ter - Gros plan Sisif. II se tourne légèrement et regarde.

2466 - Paysage qui défile (vu de la cabine).

2466 bis - Gros plan Sisif. II regarde à nouveau de face.

2467 - Plongée. Bielles en action.

(Coupure dans la copie examinée)

2477 - Locomotive vue de face amorçant un tournant. Travelling arrière. 
2478 - Gros plan. Sisif. II regarde.

2478 bis - Paysage qui défile.

2479 - Gros plan. Sisif.

2479 bis - Locomotive vue de face. Elle passe sous un pont. Travelling arrière.

2480 - Rails qui défilent.

2480 bis - Bielles en action.

2480 ter - Locomotive vue de face. Travelling arrière.

2481 - Rails qui défilent.

2482 - Paysage qui défile.

2482 bis - Locomotive de face. Travelling arrière.

2485 - Gros plan. Sisif de face.

- Locomotive de face. Travelling arrière.

- Gros plan Sisif de face. II se tourne.

- Locomotive de face.

- Sisif de profil.

- Locomotive de face.

- Sisif de profil. II se tourne de face.

- Locomotive de face.

- Sisif de face.

- Locomotive de face.

- Sisif de face. il regarde.

- Locomotive de face.

- Paysage qui défile.

- La voie qui se termine sur un butoir (travelling avant).

2485 bis - Plan d'ensemble. La locomotive heurte violemment le butoir. Deux photogrammes teintés en orange.

2485 ter - Plan moyen. La locomotive vue de face. Fumée.

2486 - Plan rapproché. Sisif contre le tas de charbon, yeux clos. II se redresse.

2487 - Plan moyen. La locomotive vue de face.

2488 - Plan rapproché. Sisif au milieu de la fumée.

2489 - Plan d'ensemble. La locomotive vue de l'arrière. Fumée.

2490 - Plan rapproché. On commence à mieux apercevoir Sisif.

2491 - Plan moyen. La locomotive vue de face.

2492 - Plan rapproché. Sisif ouvre les yeux, regarde autour de lui.

2493 - Plan moyen. Locomotive vue de profil. Beaucoup de fumée autour du cylindre éventré.

2494 - Plan rapproché. Mâchefer devant la cabine d'aiguillage.

2495 - Plan d'ensemble. La locomotive vue de trois-quart.
2496 - Plan rapproché. Sisif regarde autour de lui.

2497 - Gros plan. Cylindre droit crevé, laissant échapper la vapeur.

2498 - Plan rapproché Sisif. II regarde fixement vers la gauche, s'avance.

2499 - Plan moyen. Locomotive vue de l'avant. Boîte à fumée ouverte. Butoir démoli. Des fleurs. Fumée.

2500 - Plan rapproché. Locomotive vue de l'avant. Fleurs.

$\mathbf{2 5 0 0}$ bis - Plan rapproché Sisif. II regarde intensément vers la gauche et sort du champ.

2501 - Plan rapproché. Avant de la locomotive, vu de profil, enfoncé dans la terre. Pièces de machine. Fleurs.

2502 - Plan d'ensemble. Locomotive de trois quarts. Sisif s'avance en se tenant au tablier.

2503 - Plan rapproché avant locomotive de profil. Sisif émerge de dessous, regarde la locomotive, puis autour de lui.

2507 [sic] - Plan rapproché. Avant locomotive. Boîte à fumée ouverte. Pièces détachées. Fleurs.

- Gros plan. Une grosse marguerite apparaît.

2508 - Plan rapproché. Sisif, tête levée, regarde sa locomotive.

sous-titre: "L'Agonie de Norma Compound."

2513 [sic] - Plan d'ensemble. Ombre de la locomotive sur le sol. On aperçoit Sisif devant.

2514 - Plan moyen. Sisif accroupi devant la boîte à fumée. II regarde tristement sa locomotive.

2515 - Plan rapproché. Sisif accroupi de face. II se tourne, lève la tête. 2516 - Plan rapproché. Sisif accroupi de profil. II regarde tristement sa lomotive et se penche vers elle.

2517 - Plan rapproché. Sisif se redresse.

sous-titre: "Tu ne m'en veux pas, Norma?"

2523 [sic] - Suite du plan précédent.

2524 - Cheminée de la locomotive. On lit dans la fumée: NON... SISIF. 2525 - Plan américain Sisif de face assis en tailleur, les yeux levés. II sanglote.

2527 [sic] - Plan rapproché Sisif. Suite du même mouvement.

sous-titre: "Adieu, ma vieille!..."

2528 - Suite du plan précédent.

2529 - Gros plan cheminée. La fumée cesse de sortir.

sous-titre: "Norma Compound était morte."

2530 - Plan moyen Sisif couché contre sa lomotive, bras repliés sur lui. Fermeture à l'iris sur son visage. 